\title{
Study on the relationship between sex hormone changes and erectile dysfunction in patients with chronic prostatitis/chronic pelvic pain syndrome
}

\author{
Donglai Fan $^{1 \#}$, Weipu Mao ${ }^{2 \#}$, Guangchun Wang ${ }^{1 \#}$, Heng Shi ${ }^{3}$, Zonglin Wu ${ }^{4}$, Jinbo Xie ${ }^{1}$, Lei Yin ${ }^{1}$, \\ Tianyuan Xu' ${ }^{1}$, Keyi Wang ${ }^{1}$, Bo Peng ${ }^{1,3,4}$
}

${ }^{1}$ Department of Urology, Shanghai Tenth People's Hospital, School of Medicine, Tongji University, Shanghai, China; ${ }^{2}$ Department of Urology, Affiliated Zhongda Hospital of Southeast University, Nanjing, China; ${ }^{3}$ Department of Urology, Shanghai Tenth People's Hospital, Nanjing Medical University, Nanjing, China; ${ }^{4}$ Department of Urology, People's Hospital of Putuo District, School of Medicine, Tongji University, Shanghai, China

Contributions: (I) Conception and design: D Fan, H Shi, W Mao, B Peng; (II) Administrative support: T Xu, B Peng; (III) Provision of study materials or patients: L Yin, Z Wu, T Xu, J Xie; (IV) Collection and assembly of data: K Wang, Z Wu, G Wang; (V) Data analysis and interpretation: K Wang, Z Wu, G Wang; (VI) Manuscript writing: All authors; (VII) Final approval of manuscript: All authors.

\#These authors contributed equally to this work.

Correspondence to: Keyi Wang; Bo Peng. Department of Urology, Shanghai Tenth People's Hospital, School of Medicine, Tongji University, No. 301, Yanchang Road, Jing'an District, Shanghai 200072, China. Email: wangkeyi0910@163.com; pengbo6908@163.com.

Background: To investigate the relationship between serum sex hormones and erectile dysfunction (ED), changes in erectile function and sex hormones were studied in patients with chronic prostatitis/chronic pelvic pain syndrome (CP/CPPS).

Methods: A total of 171 patients with CP/CPPS who met the inclusion criteria from January 2016 to June 2019 were retrospectively analyzed. The level of patient's testosterone (TT), estradiol (E2), luteinizing hormone $(\mathrm{LH})$, follicle stimulating hormone (FSH), prolactin (PRL), premature ejaculation diagnostic tool (PEDT) score and international index of erectile function (IIEF-5) score were separately observed and compared.

Results: Among 171 eligible patients, 131 (76.61\%) cases were diagnosed as ED and 40 (23.39\%) cases were normal. Between the ED and No-ED groups, the PRL and PEDT score were statistically significant $(\mathrm{P}<0.01)$ based on the test results. ED-dependent and PEDT-dependent receiver operating characteristic (ROC) analysis and decision curve analysis (DCA) were performed on different degrees of ED to determine the predictive performance and clinical applicability. The results showed that PRL can better predict the possibility of ED in CP/CPPS patients than PEDT.

Conclusions: For CP/CPPS patients, the Prolactin level decreases as the degree of ED increases. Prolactin can be used as a predictor to better predict the possibility of ED in CP/CPPS patients.

Keywords: Prolactin; erectile dysfunction (ED); chronic prostatitis/chronic pelvic pain syndrome (CP/CPPS); predictive indicator

Submitted Apr 19, 2020. Accepted for publication Sep 18, 2020.

doi: 10.21037/apm-20-985

View this article at: http://dx.doi.org/10.21037/apm-20-985 


\section{Introduction}

The incidence of prostatitis is high and its complex symptoms seriously affect the quality of the life of patients (1). Chronic prostatitis/chronic pelvic pain syndrome (CP/CPPS) as category III is classified as aseptic prostatitis according to the National Institutes of Health (NIH) (2). As the most common type of prostatitis, it accounts for $90-95 \%$ of prostatitis diagnosis and the prevalence rate is about $15 \%$ of the male population (3). $\mathrm{CP} / \mathrm{CPPS}$ as a prostatitis seriously afflicts the healthy life of men. At present, the treatment for CP/CPPS are constantly updated. The current methods of treating CP/ CPPS include antibiotics, alpha blockers, phosphodiesterase inhibitors, $5-\alpha$ reductase inhibitors and so on (4). Additionally, physiotherapy has been shown to be effective in relieving symptoms of CP/CPPS, such as acupuncture (5), low-intensity extracorporeal shock wave therapy (LiESWT) (6) and circumcision (4).

Erectile dysfunction (ED) is defined as the sustained inability to obtain and maintain sufficient erections to get satisfactory sexual performance (7). Related studies indicate a correlation between ED and CP/CPPS, the incidence of $\mathrm{ED}$ is generally higher in patients with $\mathrm{CP} /$ CPPS (8). According to several studies, the prevalence of $\mathrm{ED}$ is $15.0 \%$ to $40.5 \%$ in CP/CPPS patients in China according to several studies (9). Relevant studies indicated that the obstruction of penile blood circulation is the pathological mechanism of ED (10). Moreover, the animal model studies we conducted have indicated that decreased levels of endothelial nitric oxide synthase (eNOS) and cyclic guanosine monophosphate (cGMP) in penile tissue may be the causes of ED in CP/CPPS (11-13).

In practice, atherosclerosis and diabetes are the most common causes of ED. Additionally, obesity, nerve damage and abnormal hormone expression are all risk factors for ED (10). The role of sex hormones in the development of ED has also been well studied; especially testosterone, prolactin, estradiol, etc. (14). For example, free testosterone is one of the predictors of ED occurrence (15). However, there is still a lack of clinical investigations for $\mathrm{CP} / \mathrm{CPPS}$ patients with ED, especially the relationship between various serum hormones and ED. We conducted a retrospective study of CP/CPPS patients to explore the effects of sex hormones on ED in CP/CPPS patients. Our investigation can better complement the mechanism of ED in CP/CPPS patients, as a supplement to verify the results of animal experiments and guide further research directions. Simultaneously, the results of our research can assist in clinical diagnosis and distinguishing distinguish different kinds types of ED for the individualized treatment. We present the following article in accordance with the MDAR reporting checklist (available at http:// dx.doi.org/10.21037/apm-20-985).

\section{Methods}

\section{Clinical materials}

From January 2016 to June 2019, a total of 326 patients with CP/CPPS were treated in our hospital, of which 171 patients met the inclusion criteria. The inclusion criteria were as follows: (I) the patients with CP/CPPS received anti-inflammatory analgesic treatment; (II) the chief complaints concluded an abnormality in the erectile function; (III) the patients signed an informed consent form; (IV) the patients completed relevant questionnaires [premature ejaculation diagnostic tool (PEDT) and international index of erectile function (IIEF-5)] and laboratory examinations. Exclusion criteria: (I) personal history of the patients included endocrine diseases such as diabetes etc.; (II) personal history of the patients included neurological and genetic disorders; (III) the diagnosis of the patients included urinary tract infection; (IV) the patients had testicular disease such as testicular torsion etc.; (V) patients' diagnosis included varicocele.

The diagnostic criteria of CP/CPPS complied in this study were as follows: (I) history of present illness had recurrent pain in the perineum, suprapubic or lumbosacral area lasting more than three months; (II) laboratory tests ruled out urinary tract infections; (III) no pathogens such as bacteria were found in expressed prostatic secretions (EPS). Simultaneously, the diagnostic criteria of ED used in our study were complied with the National Institutes of Health (NIH) criteria (https://www.niddk. nih.gov/health-information/urologic-diseases/erectiledysfunction? dkrd=hispt0409).

The detailed study design was shown in Figure 1. All procedures performed in this study were in accordance with the Declaration of Helsinki (as revised in 2013) and approved by the Ethics Committee of Shanghai Tenth People's Hospital, School of Medicine, Tongji University (SHSY-IEC-KY-4.0/18-68/01) and written informed consent was obtained from all patients or their relatives. 


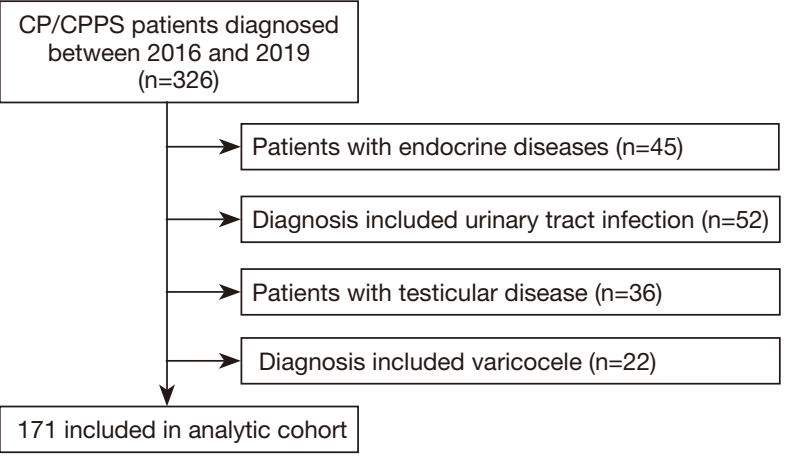

Figure 1 Study design flowchart of specific patient screening process.

Table 1 Baseline characteristics of total patients

\begin{tabular}{lc}
\hline Characteristics & Median \pm SD \\
\hline Total $(\%)$ & $38.89 \pm 8.88$ \\
Age (years) & $23.54 \pm 3.00$ \\
BMl $\left(\mathrm{kg} / \mathrm{m}^{2}\right)$ & $24.08 \pm 136.11$ \\
TT $(\mathrm{nmol} / \mathrm{L})$ & $74.41 \pm 44.10$ \\
E2 (pmol/L) & $4.99 \pm 2.69$ \\
LH (IU/L) & $5.06 \pm 4.36$ \\
FSH (IU/L) & $258.87 \pm 129.34$ \\
PRL (mIU/L) & $9.38 \pm 4.87$ \\
PEDT & $16.27 \pm 6.03$
\end{tabular}

BMI, body mass index; TT, testosterone; E2, estradiol; LH, luteinizing hormone; $\mathrm{FSH}$, follicle stimulating hormone; $\mathrm{PRL}$, prolactin; PEDT, premature ejaculation diagnostic tool; IIEF-5, international index of erectile function.

\section{Study variables}

We counted the basic information of the patient, such as age and body mass index (BMI). Simultaneously, the patients' sex hormone variables were recorded, included testosterone (TT), estradiol (E2), luteinizing hormone $(\mathrm{LH})$, follicle stimulating hormone (FSH) and prolactin (PRL). The patients received an investigation of ED related questionnaire, including PEDT and international index of erectile function (IIEF-5). All the above information was recorded by specialized researchers and kept properly. The patient information was kept by a dedicated clinical investigator to prevent information leakage. In addition, data collection and processing require the consent of all participants in this study to proceed.
According to whether suffering from ED based on the diagnostic criteria adopted in our study, the patients were divided into two groups: ED and No-ED. The degree of ED was determined by inquiring about the patient's own history of sexual intercourse and completing the investigation of the patient's confidence, satisfaction and duration of the intercourse process based on the IIEF-5. And the patients with ED were separated into three subgroups [Mild group (12 to 21), Moderate group (8 to 11) and Severe group (5 to 7)] based on the varying degrees of ED.

\section{Statistical method}

Statistical Package for the Social Sciences software (SPSS, version 20.0) was used to analyze and process the data. The chi-square test was used to analyze the clinical factors related to ED and the subgroups. Based on the statistical results, PRL was included as the target variable. The relationship between PRL and ED was analyzed by correlation test, receiver operating characteristic (ROC) and decision curve analysis (DCA) analysis. $\mathrm{P}<0.05$ indicated statistical significance. DCA was conducted using R software (v. 3.5.1, Vienna, Austria). The "rmda" package was used to construct the DCA curve plots.

\section{Results}

\section{General information of patients}

We confirmed 171 eligible patients from January 2016 to June 2019 in our medical center. Table 1 shows the baseline characteristics of total patients. Among the eligible patients, 131 cases were diagnosed as ED and 40 cases were normal. The average age and BMI of the study sample were $38.89 \pm 8.88$ years and $23.54 \pm 3.00 \mathrm{~kg} / \mathrm{m}^{2}$. Average PRL was $258.87 \pm 129.34 \mathrm{mIU} / \mathrm{L}$. The average values of other sex hormone indicators such as TT, E2, LH and FSH were $24.08 \pm 136.11 \mathrm{nmol} / \mathrm{L}, 74.41 \pm 44.10 \mathrm{pmol} / \mathrm{L}, 4.99 \pm$ $2.69 \mathrm{IU} / \mathrm{L}$ and $5.06 \pm 4.36 \mathrm{IU} / \mathrm{L}$. Average PEDT and IIEF-5 score were $9.38 \pm 4.87,16.27 \pm 6.03$ respectively.

\section{Comparison of sex hormone levels in No-ED group and ED group}

According to the IIEF-5 index, patients were divided into two groups: No-ED group (22 to 25) and ED group (5 to 21). The clinical characteristics of all patients stratified by whether suffering from ED were showed by Table 2. There 
Table 2 Clinical characteristics of all patients stratified by whether suffering from ED

\begin{tabular}{lccc}
\hline Characteristics & No-ED & ED & P value \\
\hline Total (\%) & $40(23.39)$ & $131(76.61)$ & 0.241 \\
Age (years) & $37.45 \pm 9.13$ & $39.34 \pm 8.79$ & 0.534 \\
BMI (kg/m²) & $23.28 \pm 3.15$ & $23.32 \pm 2.96$ & 0.056 \\
TT (nmol/L) & $15.16 \pm 5.34$ & $13.21 \pm 5.71$ & 0.607 \\
E2 (pmol/L) & $77.31 \pm 43.89$ & $73.44 \pm 44.29$ & 0.528 \\
LH (IU/L) & $4.76 \pm 1.38$ & $5.06 \pm 2.98$ & 0.691 \\
FSH (IU/L) & $4.82 \pm 2.46$ & $5.14 \pm 4.79$ & $<0.001$ \\
PRL (mIU/L) & $374.18 \pm 135.92$ & $223.67 \pm 104.85$ & 0.003 \\
PEDT & $7.43 \pm 4.76$ & $9.98 \pm 4.76$ & $<0.001$ \\
IIEF-5 & $23.25 \pm 0.90$ & $14.14 \pm 5.26$ & \\
\hline
\end{tabular}

BMI, body mass index; TT, testosterone; E2, estradiol; LH, luteinizing hormone; FSH, follicle stimulating hormone; PRL, prolactin; PEDT, premature ejaculation diagnostic tool; IIEF-5, international index of erectile function.

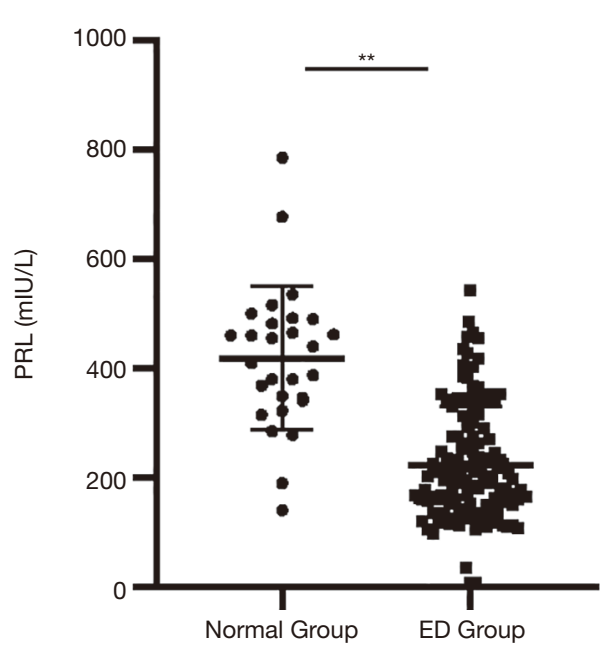

Figure 2 The scatter plot of PRL expression levels in No-ED group and ED group $\left({ }^{* *} \mathrm{P}<0.01\right)$. $\mathrm{PRL}$, prolactin; $\mathrm{ED}$, erectile dysfunction.

was no significant difference in age, BMI, testosterone, estradiol, $\mathrm{LH}$ and FSH $(\mathrm{P}>0.05)$. The value of PRL $(374.18 \pm 135.92 \mathrm{mIU} / \mathrm{L})$ in No-ED group was higher than it $(223.67 \pm 104.85 \mathrm{mIU} / \mathrm{L})$ in ED group. And the comparison was statistically significant (Figure 2). The values of PEDT score were $7.43 \pm 4.76$ in No-ED group and $9.98 \pm 4.76$ in ED group. Similarly, the values of IIEF-5 were $23.25 \pm 0.90$ (NoED group) and $14.14 \pm 5.26$ (ED group).

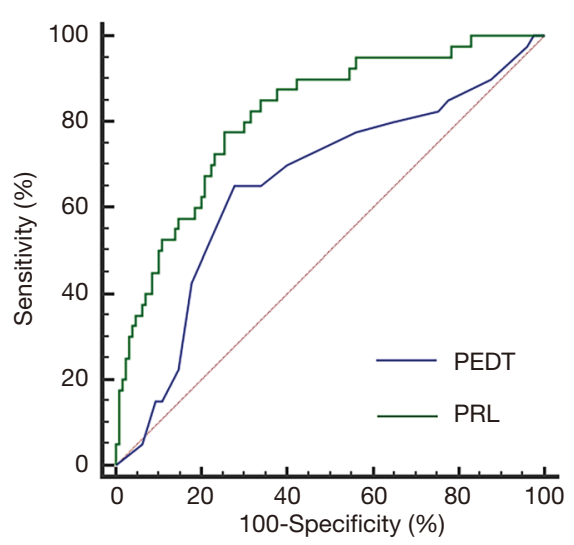

Figure 3 ROC curve for PRL-dependent and PEDT-dependent. ROC, receiver operating characteristic; PRL, prolactin; PEDT, premature ejaculation diagnostic tool.

\section{Effect of PRL in predicting ED}

Analyzing the relationship between PRL and ED by correlation test. The Kendall correlation coefficient (r) was $0.381(\mathrm{P}<0.01)$. ED-dependent and PEDT-dependent receiver operating characteristic (ROC) analysis showed that the area under curve (AUC) of PRL and PEDT for predicting ED were 0.817 and 0.657 (Figure 3). DCA curve was used to assess whether PRL would help with clinical treatment strategies (Figure 4). In our study, when the threshold probability varied from 0 to 1 , according to the 
DCA, "PRL" achieved the most net benefit compared with "treat all" strategy, "treat none" strategy, and PEDT. It was found that PRL can better predict the possibility of ED in CP/CPPS patients than PEDT. And the cut-off points for predicting ED was $275.9 \mathrm{mIU} / \mathrm{L}$.

\section{Comparison of sex hormone levels in different ED group}

According to the IIEF-5 index, patients were divided into three groups: Mild group (12 to 21), Moderate group (8

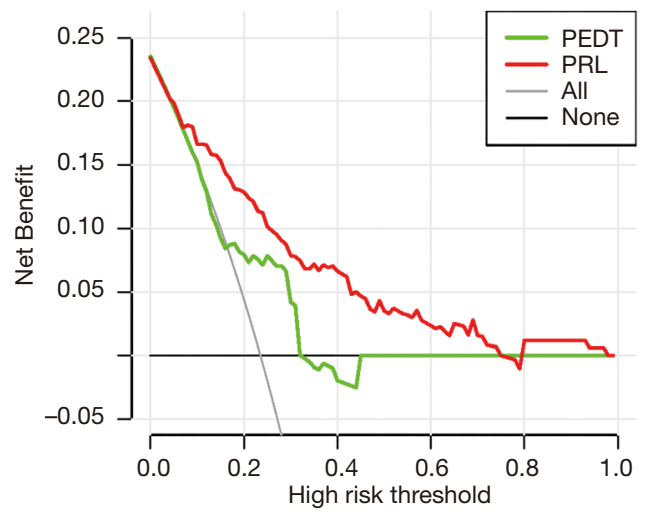

Figure 4 DCA curve of clinical use assessment of the PRL and the PEDT in the training cohort. The net benefit is shown on the $y$-axis and the threshold probability is shown on the $\mathrm{X}$-axis. DCA, decision curve analysis; PRL, prolactin; PEDT, premature ejaculation diagnostic tool. to 11 ) and Severe group (5 to 7). Table 3 shows the clinical characteristics of all patients stratified by the severity of ED. PRL, estradiol and PEDT had significant differences between different groups $(\mathrm{P}<0.05$, Figure $5 A)$. The values of PRL in different groups were $245.21 \pm 106.84 \mathrm{mIU} / \mathrm{L}$ (Mild group), $183.98 \pm 58.08 \mathrm{mIU} / \mathrm{L}$ (Moderate group) and $134.39 \pm 28.98 \mathrm{mIU} / \mathrm{L}$ (Severe group) respectively. The scatter diagram between PRL and ED was drawn

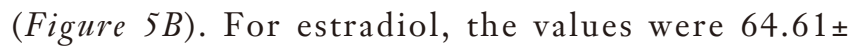
$40.74 \mathrm{pmol} / \mathrm{L}$ (Mild group), 76.59 $\pm 39.07 \mathrm{pmol} / \mathrm{L}$ (Moderate group) and $120.47 \pm 42.74 \mathrm{pmol} / \mathrm{L}$ (Severe group) respectively.

\section{Discussion}

As the most common type of CP, CP/CPPS seriously affects men's health (4). ED is one of the functional diseases that afflict middle-aged men (16). Previous studies have reported that CP/CPPS is associated with ED $(8,9)$. And the results in animal models also indicated that CP/CPPS was one of the causes of ED $(2,11,12)$. In addition, abnormal levels of sex hormone are important causes of ED and testosterone therapy or phosphodiesterase type 5 inhibitors have been proven effective in treating $\mathrm{ED}(17,18)$. The aim of our study was to investigate the relationship between sex hormone changes and ED in CP/CPPS patients.

Low androgen levels or abnormalities in androgen receptors were partially account for ED (19). Testosterone as the most important androgen in men, plays an effective role in the treatment of $\operatorname{ED}(20,21)$. About $44 \%$ of

Table 3 Clinical characteristics of all patients stratified by the severity of ED

\begin{tabular}{|c|c|c|c|c|}
\hline Characteristics & Mild & Moderate & Severe & $P$ value \\
\hline Total (\%) & $92(70.77)$ & 22 (16.92) & 16 (12.31) & \\
\hline Age (years) & $38.61 \pm 8.53$ & $40.45 \pm 9.46$ & $41.63 \pm 9.26$ & 0.357 \\
\hline BMI $\left(\mathrm{kg} / \mathrm{m}^{2}\right)$ & $23.41 \pm 2.71$ & $23.79 \pm 3.74$ & $24.63 \pm 3.23$ & 0.305 \\
\hline TT (nmol/L) & $13.05 \pm 5.87$ & $14.13 \pm 4.90$ & $12.87 \pm 5.99$ & 0.707 \\
\hline LH (IU/L) & $4.90 \pm 3.03$ & $6.02 \pm 3.51$ & $4.56 \pm 1.29$ & 0.229 \\
\hline FSH (IU/L) & $4.97 \pm 5.34$ & $5.93 \pm 3.29$ & $5.06 \pm 3.12$ & 0.704 \\
\hline PRL (mlU/L) & $245.21 \pm 106.84$ & $183.98 \pm 58.08$ & $134.39 \pm 28.98$ & $<0.001$ \\
\hline PEDT & $9.14 \pm 4.74$ & $12.95 \pm 3.37$ & $10.50 \pm 5.11$ & 0.003 \\
\hline
\end{tabular}

BMI, body mass index; TT, testosterone; E2, estradiol; LH, luteinizing hormone; FSH, follicle stimulating hormone; PRL, prolactin; PEDT, premature ejaculation diagnostic tool; IIEF-5, international index of erectile function. 

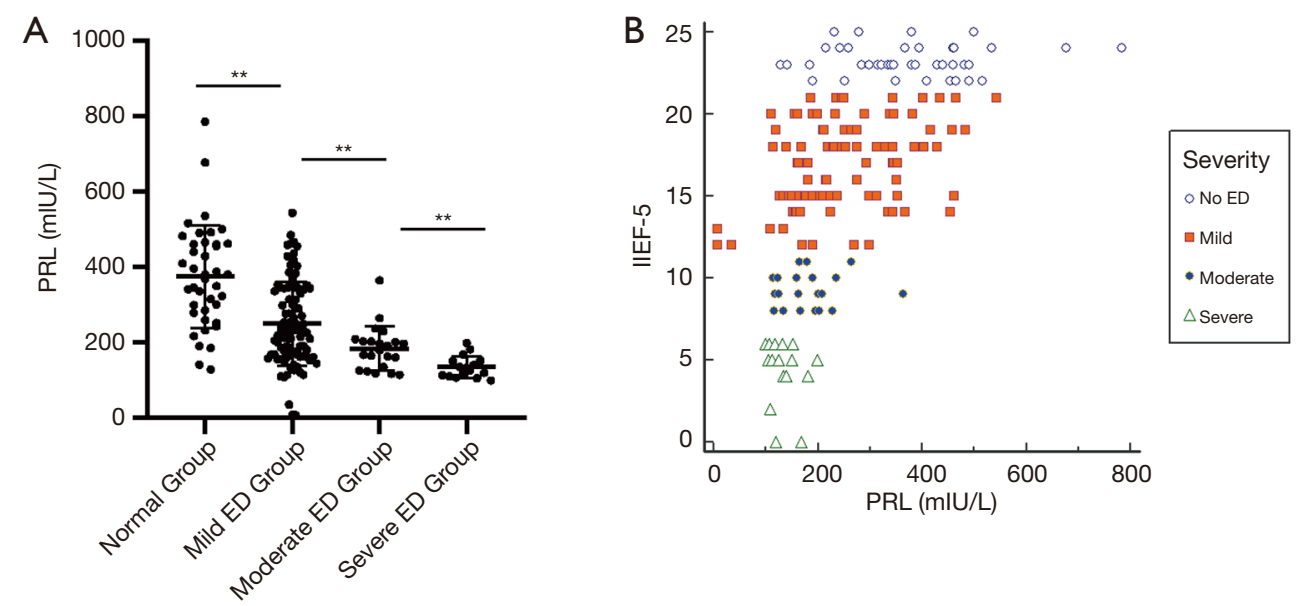

Figure 5 PPRL expression levels in different degrees of ED grouping. (A) The scatter plot of PRL expression $\left({ }^{* *} \mathrm{P}<0.01\right)$; (B)The scatter diagram between PRL and ED. PRL, prolactin; PEDT, premature ejaculation diagnostic tool; ED, erectile dysfunction.

testosterone combined to serum sex hormone-binding globulin (SHBG) to form testosterone without biological activity and bioavailable testosterone (Bio-T) did affect sexual function (22). Martinez-Jabaloyas et al. (23) found that the total testosterone (TT) levels were not related to sexual function in men with ED. And it has been reported that free testosterone (FT) has a predictive effect on the occurrence of ED after radical prostatectomy (15). Our study found that comparison between groups in CP/CPPS patients were not statistically significant $(\mathrm{P}>0.05)$.

Hypothalamic-pituitary-gonadal axis (HPGA) plays an important role in male sexual function maturation and maintenance (24). $\mathrm{LH}$ and $\mathrm{FSH}$, as the important components in the axis, their functions were self-evident. They can promote the maturation of follicles and more researches are now focused on female reproduction. FSH worked on male sperm maturation (25). We can distinguish between primary and secondary testosterone deficiency (TD) by detecting LH levels (14). It has been reported that the use of luteinizing hormone-releasing hormone (LRH) to promote $\mathrm{LH}$ release also had a certain promoting effect on normal male sexual function (26). However, there are still no related studies found that there is a correlation with ED currently (27). The results of our study also indicated that they were not related to $\mathrm{ED}(\mathrm{P}>0.05)$.

Estradiol as a kind of estrogen promoted female secondary sexual development and sexual organ maturation. It will combine SHBG to affect testosterone expression in males and further affect sexual function (28). Related reports had confirmed that high estradiol was positively correlated with erectile function $(29,30)$. And the testosterone : estrogen ratio was found to be associated with sexual function $(31,32)$. Increased ratio of estrogen: testosterone in animal studies indicated a negative effect on sexual function (33). Mancini et al. (34) found serum E2 positively correlated with severity of ED secondary to venous leak. At the same time, elevated estradiol was a contributing factor to PRL. It could directly act on the pituitary to promote PRL release; increased the sensitivity of the anterior pituitary to PRL regulatory factors (35). As we found, estradiol levels were positively correlated with ED levels $(\mathrm{P}<0.05)$; but it didn't make sense for the diagnosis of ED. This may be due to the lack of our sample size. Prospective study of multicenter participation is urgently needed to further study its role in sexual function.

PRL, as one part of HGPA had been reported in studies, was related with male sexual function $(14,36)$. Many related studies had reported that PRL was associated with libido $(37,38)$. The low libido exhibited by hyperprolactinemia was mainly related to the inhibition of HGPA $(39,40)$. This indirectly indicated that low PRL had the potential to cause sexual dysfunction. There is also evidence that low PRL levels also influence sexual function. Related epidemiological surveys indicate that low PRL is associated with impaired penile blood flow and ED $(41,42)$. Our findings also proved these relationship PRL levels were negatively correlated with $\mathrm{ED}(\mathrm{P}<0.05)$. Treatment of hyperprolactinemia in men with reduced PRL medication could restore libido; this 
result was not available in testosterone therapy $(43,44)$. It is speculated that PRL can directly affect sexual function. This had also been verified in animal experiments (45).

At present, the specific mechanism to explain this association is not clear. The association between low PRL and ED can be explained by the central 5-hydroxytryptamine (5-HT) reduction (36). Studies had confirmed that 5-HT is the stimulating factor of PRL (46). Researches in animal models had also shown that 5-HT is positively correlated with PRL (47). There were evidences demonstrated that agonists of 5-HT could induce erection in animal models and the antagonists could prevent the dopamine (DA)-induced erection (48). In summary, the relationship between low PRL and ED could be explained by a reduction of central 5-HT. However, there is still no further experiment to prove this hypothesis.

The relationship between premature ejaculation (PE) and ED has been reported in related studies $(49,50)$. PEDT score as a method of evaluating PE can also predict ED. Our study found that patients with ED have lower PEDT scores which further corroborated the relevant findings. But its ROC analysis result showed that its prediction accuracy was lower than PRL (0.657 vs. 0.817). This result further demonstrated the feasibility of PRL as a predictor. We also used a DCA curve to assess whether PRL would help with clinical treatment strategies. DCA showed that the PRL outperformed the PEDT across a wide range of threshold probabilities, which revealed that the clinical parameters added incremental value to ED risk evaluation in both the primary and external cohorts.

Our study initially revealed the predictive role of sex hormones in developing ED in patients with CPP/CPPS. As a retrospective study, this study had various limitations. Firstly, the small sample size was the point that cannot be ignored. Secondly, statistical bias is an important deficiency of retrospective research. The selection bias caused by subjective factors in data statistics and processing was also a defect in our study. Thirdly, the types of clinical factors included in our study were less and needed to be further expanded. Additionally, the patients' information included in this study was only from one clinical center and may not be representative. There is still a lack of relevant multicenter prospective clinical trials to further demonstrate the results which is also the focus of our future work.

\section{Conclusions}

Patients with CP/CPPS are more susceptible to ED than healthy people. Serum sex hormone abnormalities are an important cause of ED, but no studies have been conducted in patients with CP/CPPS. Our retrospective study of the relationship between sex hormones and ED in patients with CP/CPPS found that routine examination of PRL can be used as a predictor of ED; and its level was positively correlated with ED. Future prospective studies are needed to validate its value as a predictive indicator.

\section{Acknowledgments}

Funding: This work was supported by grants from Shanghai Science and Technology Commission (Grant No. 18140900302), the National Natural Science Foundation of China (Grant No. 81870517) to Bo Peng and the Climbing Talent Projects of Shanghai Tenth People's Hospital (No. 2018SYPDRC046) and the Fundamental Research Funds for the Central Universities (No. 22120180586) to Tianyuan $\mathrm{Xu}$.

\section{Footnote}

Reporting Checklist: The authors have completed the MDAR reporting checklist. Available at http://dx.doi.org/10.21037/ apm-20-985

Data Sharing Statement: Available at http://dx.doi. org/10.21037/apm-20-985

Conflicts of Interest: All authors have completed the ICMJE uniform disclosure form (available at http://dx.doi. org/10.21037/apm-20-985). The authors have no conflicts of interest to declare.

Ethical Statement: The authors are accountable for all aspects of the work in ensuring that questions related to the accuracy or integrity of any part of the work are appropriately investigated and resolved. All procedures performed in this study were in accordance with the Declaration of Helsinki (as revised in 2013) and approved by the Ethics Committee of Shanghai Tenth People's Hospital, School of Medicine, Tongji University (SHSYIEC-KY-4.0/18-68/01) and written informed consent was obtained from all patients or their relatives.

Open Access Statement: This is an Open Access article distributed in accordance with the Creative Commons Attribution-NonCommercial-NoDerivs 4.0 International 
License (CC BY-NC-ND 4.0), which permits the noncommercial replication and distribution of the article with the strict proviso that no changes or edits are made and the original work is properly cited (including links to both the formal publication through the relevant DOI and the license). See: https://creativecommons.org/licenses/by-nc-nd/4.0/.

\section{References}

1. Liu Y, Mikrani R, Xie D, et al. Chronic prostatitis/ chronic pelvic pain syndrome and prostate cancer: study of immune cells and cytokines. Fundam Clin Pharmacol 2020;34:160-72.

2. $\mathrm{Hu} \mathrm{Y}, \mathrm{Niu} \mathrm{X}$, Wang $\mathrm{G}$, et al. Chronic prostatitis/chronic pelvic pain syndrome impairs erectile function through increased endothelial dysfunction, oxidative stress, apoptosis, and corporal fibrosis in a rat model. Andrology 2016;4:1209-16.

3. Murphy SF, Schaeffer AJ, Thumbikat P. Immune mediators of chronic pelvic pain syndrome. Nat Rev Urol 2014;11:259-69.

4. Appiya Santharam M, Khan FU, Naveed M, et al. Interventions to chronic prostatitis/Chronic pelvic pain syndrome treatment. Where are we standing and what's next? Eur J Pharmacol 2019;857:172429.

5. Wazir J, Ullah R, Li S, et al. Efficacy of acupuncture in the treatment of chronic prostatitis-chronic pelvic pain syndrome: a review of the literature. Int Urol Nephrol 2019;51:2093-106.

6. Yuan P, Ma D, Zhang Y, et al. Efficacy of low-intensity extracorporeal shock wave therapy for the treatment of chronic prostatitis/chronic pelvic pain syndrome: A systematic review and meta-analysis. Neurourol Urodyn 2019;38:1457-66.

7. Meldrum DR, Morris MA, Gambone JC, et al. Aging and erectile function. Aging Male 2019. [Epub ahead of print].

8. Zhao Z, Xuan X, Zhang J, et al. A prospective study on association of prostatic calcifications with sexual dysfunction in men with chronic prostatitis/chronic pelvic pain syndrome (CP/CPPS). J Sex Med 2014;11:2528-36.

9. Chung SD, Keller JJ, Lin HC. A case-control study on the association between chronic prostatitis/chronic pelvic pain syndrome and erectile dysfunction. BJU Int 2012;110:726-30.

10. Najari BB, Kashanian JA. Erectile Dysfunction. JAMA 2016;316:1838-

11. Huang T, Wang G, Hu Y, et al. Structural and functional abnormalities of penile cavernous endothelial cells result in erectile dysfunction at experimental autoimmune prostatitis rat. J Inflamm (Lond) 2019;16:20.

12. Huang TR, Li W, Peng B. Correlation of inflammatory mediators in prostatic secretion with chronic prostatitis and chronic pelvic pain syndrome. Andrologia 2018. doi: 10.1111/and.12860.

13. Wang XJ, Xia LL, Xu TY, et al. Changes in erectile organ structure and function in a rat model of chronic prostatitis/ chronic pelvic pain syndrome. Andrologia 2016;48:243-51.

14. Sansone A, Romanelli F, Gianfrilli D, et al. Endocrine evaluation of erectile dysfunction. Endocrine 2014;46:423-30.

15. Li T, Sun X, Chen L. Free testosterone value before radical prostatectomy is related to oncologic outcomes and post-operative erectile function. BMC Cancer 2019;19:87.

16. Mobley DF, Khera M, Baum N. Recent advances in the treatment of erectile dysfunction. Postgrad Med J 2017;93:679-85.

17. Corona G, Rastrelli G, Morgentaler A, et al. Meta-analysis of Results of Testosterone Therapy on Sexual Function Based on International Index of Erectile Function Scores. Eur Urol 2017;72:1000-11.

18. Drobnis EZ, Nangia AK. 5alpha-Reductase Inhibitors (5ARIs) and Male Reproduction. Adv Exp Med Biol 2017;1034:59-61.

19. Coward RM, Stetter C, Kunselman A, et al. Fertility Related Quality of Life, Gonadal Function and Erectile Dysfunction in Male Partners of Couples with Unexplained Infertility. J Urol 2019;202:379-84.

20. Neychev V, Mitev V. Pro-sexual and androgen enhancing effects of Tribulus terrestris L.: Fact or Fiction. J Ethnopharmacol 2016;179:345-55.

21. Muneer A, Kalsi J, Nazareth I, et al. Erectile dysfunction. Bmj 2014;348:g129.

22. Goldman AL, Bhasin S, Wu FCW, et al. A Reappraisal of Testosterone's Binding in Circulation: Physiological and Clinical Implications. Endocr Rev 2017;38:302-24.

23. Martinez-Jabaloyas JM, Queipo-Zaragoza A, PastorHernandez F, et al. Testosterone levels in men with erectile dysfunction. BJU Int 2006;97:1278-83.

24. Lee DS, Choi JB, Sohn DW. Impact of Sleep Deprivation on the Hypothalamic-Pituitary-Gonadal Axis and Erectile Tissue. J Sex Med 2019;16:5-16.

25. Tamburino L, La Vignera S, Tomaselli V, et al. The -29G/ A FSH receptor gene polymorphism is associated with higher FSH and LH levels in normozoospermic men. J Assist Reprod Genet 2017;34:1289-94.

26. Evans IM, Distiller LA. Effects of luteinizing hormone- 
releasing hormone on sexual arousal in normal men. Arch Sex Behav 1979;8:385-95.

27. Zhang XB, Lin QC, Zeng HQ, et al. Erectile Dysfunction and Sexual Hormone Levels in Men With Obstructive Sleep Apnea: Efficacy of Continuous Positive Airway Pressure. Arch Sex Behav 2016;45:235-40.

28. Rosner W. Free estradiol and sex hormone-binding globulin. Steroids 2015;99:113-6.

29. Srilatha B, Adaikan PG. Endocrine milieu and erectile dysfunction: is oestradiol-testosterone imbalance, a risk factor in the elderly? Asian J Androl 2011;13:569-73.

30. Zuniga KB, Margolin EJ, De Fazio A, et al. The association between elevated serum oestradiol levels and clinically significant erectile dysfunction in men presenting for andrological evaluation. Andrologia 2019;51:e13345.

31. Castelló-Porcar AM, Martínez-Jabaloyas JM. Testosterone/estradiol ratio, is it useful in the diagnosis of erectile dysfunction and low sexual desire? Aging Male 2016;19:254-8.

32. Wu F, Chen T, Mao S, et al. Levels of estradiol and testosterone are altered in Chinese men with sexual dysfunction. Andrology 2016;4:932-8.

33. Adaikan PG, Srilatha B. Oestrogen-mediated hormonal imbalance precipitates erectile dysfunction. Int J Impot Res 2003;15:38-43.

34. Mancini A, Milardi D, Bianchi A, et al. Increased estradiol levels in venous occlusive disorder: a possible functional mechanism of venous leakage. Int J Impot Res 2005;17:239-42.

35. Aquino NSS, Araujo-Lopes R, Henriques PC, et al. alpha-Estrogen and Progesterone Receptors Modulate Kisspeptin Effects on Prolactin: Role in EstradiolInduced Prolactin Surge in Female Rats. Endocrinology 2017;158:1812-26.

36. Rastrelli G, Corona G, Maggi M. The role of prolactin in andrology: what is new? Rev Endocr Metab Disord 2015;16:233-48.

37. Krysiak R, Drosdzol-Cop A, Skrzypulec-Plinta V, et al. Sexual function and depressive symptoms in young women with elevated macroprolactin content: a pilot study. Endocrine 2016;53:291-8.

38. An FR, Yang R, Wang ZM, et al. Hyperprolactinemia, prolactin-related side effects and quality of life in Chinese psychiatric patients. Compr Psychiatry 2016;71:71-6.

39. Rastrelli G, Carter EL, Ahern T, et al. Development of and Recovery from Secondary Hypogonadism in Aging Men: Prospective Results from the EMAS. J Clin
Endocrinol Metab 2015;100:3172-82.

40. Buvat J, Maggi M, Gooren L, et al. Endocrine aspects of male sexual dysfunctions. J Sex Med 2010;7:1627-56.

41. Corona G, Mannucci E, Jannini EA, et al. Hypoprolactinemia: a new clinical syndrome in patients with sexual dysfunction. J Sex Med 2009;6:1457-66.

42. Corona G, Wu FC, Rastrelli G, et al. Low prolactin is associated with sexual dysfunction and psychological or metabolic disturbances in middle-aged and elderly men: the European Male Aging Study (EMAS). J Sex Med 2014;11:240-53.

43. Prescott RW, Johnston DG, Kendall-Taylor P, et al. Hyperprolactinaemia in men-response to bromocriptine therapy. Lancet 1982;1:245-8.

44. Carter JN, Tyson JE, Tolis G, et al. Prolactin-screening tumors and hypogonadism in 22 men. N Engl J Med 1978;299:847-52.

45. Cruz-Casallas PE, Nasello AG, Hucke EE, et al. Dual modulation of male sexual behavior in rats by central prolactin: relationship with in vivo striatal dopaminergic activity. Psychoneuroendocrinology 1999;24:681-93.

46. Clemens JA, Sawyer BD, Cerimele B. Further evidence that serotonin is a neurotransmitter involved in the control of prolactin secretion. Endocrinology 1977;100:692-8.

47. Lindell SG, Suomi SJ, Shoaf S, et al. Salivary prolactin as a marker for central serotonin turnover. Biol Psychiatry 1999;46:568-72.

48. McDougall SA, Rios JW, Apodaca MG, et al. Effects of dopamine and serotonin synthesis inhibitors on the ketamine-, d-amphetamine-, and cocaine-induced locomotor activity of preweanling and adolescent rats: sex differences. Behav Brain Res 2020;379:112302.

49. Corona G, Rastrelli G, Limoncin E, et al. Interplay Between Premature Ejaculation and Erectile Dysfunction: A Systematic Review and Meta-Analysis. J Sex Med 2015;12:2291-300.

50. Brody S, Weiss P. Erectile dysfunction and premature ejaculation: interrelationships and psychosexual factors. J Sex Med 2015;12:398-404.

Cite this article as: Fan D, Mao W, Wang G, Shi H, Wu Z, Xie J, Yin L, Xu T, Wang K, Peng B. Study on the relationship between sex hormone changes and erectile dysfunction in patients with chronic prostatitis/chronic pelvic pain syndrome. Ann Palliat Med 2021;10(2):1739-1747. doi: 10.21037/apm-20-985 